\title{
Comparison of colonoscopy after colonic diverticulitis and positive fecal immunochemical tests for the detection of colorectal neoplasia
}

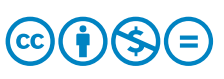

\author{
Authors \\ Dai Nakamatsu, Tsutomu Nishida, Shinji Kuriki, Li-sa Chang, Kazuki Aochi, Emi Meren, Tatsuya Sakamoto, Ryo \\ Tomita, Yu Higaki, Naoto Osugi, Aya Sugimoto, Kei Takahashi, Kaori Mukai, Kengo Matsumoto, Shiro Hayashi, \\ Sachiko Nakajima, Masashi Yamamoto, Koji Fukui, Masami Inada
}

Institution

Department of Gastroenterology, Toyonaka Municipal Hospital, Osaka, Japan

submitted 30.6.2020

accepted after revision $\quad 2.11 .2020$

Bibliography

Endoscopy International Open 2021; 09: E331-E337

DOI 10.1055/a-1333-1108

ISSN 2364-3722

(c) 2021. The Author(s).

This is an open access article published by Thieme under the terms of the Creative Commons Attribution-NonDerivative-NonCommercial License, permitting copying and reproduction so long as the original work is given appropriate credit. Contents may not be used for commecial purposes, or adapted, remixed, transformed or built upon. (https://creativecommons.org/licenses/by-nc-nd/4.0/)

Georg Thieme Verlag KG, Rüdigerstraße 14,

70469 Stuttgart, Germany

Corresponding author

Tsutomu Nishida, Ph, MD, Department of Gastroenterology, Toyonaka Municipal Hospital, 4-14-1 Shibahara, Toyonaka, Osaka 560-8565, Japan

Fax: +81-6-6858-3531

tnishida.gastro@gmail.com

\section{ABSTRACT}

Background and study aims The relationship between acute colonic diverticulitis and colorectal cancer (CRC) is unclear, but colonoscopy is recommended to exclude malignancy. We compared the detection rates for colorectal neoplasia in patients with colonic diverticulitis and asymptomatic patients who had positive fecal immunochemical tests (FITs).

Patients and methods In total, 282 patients with acute colonic diverticulitis were hospitalized in our hospital from February 2011 to December 2019. Of them, 143 patients with diverticulitis and 1819 with positive FITs patients during the same period underwent colonoscopy without a prior colonoscopy within 5 years. We retrospectively compared these patients in terms of the invasive CRC rate, advanced neoplasia detection rate (ANDR), adenoma detection rate (ADR), and polyp detection rate (PDR).

Results Compared to the diverticulitis group, the FIT-positive group had a significantly higher CRC rate ( 0 vs $2.7 \%, P=$ 0.0061 ), ANDR (5.6 vs. $14.0 \%, P=0.0017)$, ADR (19.6 vs. $53.2 \%, P<.0001)$, and PDR (44.1 vs. $91.0 \%, P<.0001)$. Using 1:1 propensity score matching based on age and sex, we obtained 276 matched patients in both groups. After matching, no difference was found in the CRC rate (0 vs $0.7 \%$ ) or ANDR (5.8 vs $7.3 \%$ ) between groups, but the ADR and PDR were significantly higher in the FIT-positive group (20.3 vs $43.5 \%, P<.0001 ; 45.7 \%$ vs $86.2 \%, P<.0001)$. Conclusion Patients with acute diverticulitis had lower ADRs and PDRs than patients with positive FITs.

\section{Introduction}

In recent years, with the westernization of eating habits and aging population in Japan, the prevalence of colonic diverticulum has increased, and the opportunity to treat colonic diverticulosis has increased worldwide. Colonic diverticulitis, which is one of the complications of colonic diverticulosis, is diagnosed by clinical symptoms and imaging, such as computed tomography $(C T)$, and colorectal cancer (CRC) is one of the differential diagnoses. The American Gastroenterological Association (AGA) guidelines recommend that patients undergo a colonoscopy 4 to 8 weeks after an event of diverticulitis if they have not recently undergone colonoscopy [1]. This recommendation is based on some observational studies of patients with diverticulitis diagnosed by imaging and subsequent colonoscopy. According to the AGA technical review, 1 in 67 patients (1.5\%) initially diagnosed with diverticulitis are estimated to be misdiagnosed with CRC on follow-up colonoscopy [2]. However, it is un- 
clear whether there is a relationship between diverticulitis and CRC in the Japanese population [3]. In a previous study, it was reportedly uncertain whether patients with colon diverticulitis have a higher rate of CRC than the general population $[4,5]$. The 2017 Japan Gastroenterological Association (JGA) guidelines also recommend performing a colonoscopy to exclude malignancies other than colonic diverticulosis [3]. However, whether it is appropriate to have a similar strategy in Japan is unclear because diverticular disease in the right colon is more prevalent in Japan than in Western countries [3]. In addition, the current guidelines do not specifically indicate the risk of CRC in patients with acute diverticulitis. In the present study, we investigated the impact of initial colonoscopy after an acute colonic diverticulitis event on the detection of colorectal neoplasms in comparison to the impact of colonoscopy due to positive fecal immunochemical tests (FITs).

\section{Patients and methods}

There were 297 consecutive patients with suspected acute colonic diverticulitis diagnosed by computed tomography (CT) who were hospitalized in the Toyonaka Municipal Hospital from February 2011 to December 2019. Acute diverticulitis was diagnosed based on CT images showing bowel wall thickening and fat stranding consistent with diverticulitis, abdominal pain and elevated C-reactive protein levels and white blood cell counts. We enrolled diverticulitis patients with an initial colonoscopy or no colonoscopy history within 5 years as the diverticulitis group, and all of these patients underwent colonoscopy 24 weeks after an event of diverticulitis except for those who underwent surgery. During the same period, we enrolled asymptomatic patients who were referred to our hospital due to positive FIT results from a CRC screening program as a control group. Japan introduced a CRC screening program with FIT in 1992. The patients in the control group showed at least one positive result with a cut-off value of $100 \mathrm{ng} / \mathrm{mL}$ in a 1 -day test or 2-day test from the screening program and visited our hospital for colonoscopy. Then, we retrospectively evaluated and compared patients with acute diverticulitis and FIT-positive patients in terms of their CRC neoplasia rates.

The present study was conducted in accordance with the Declaration of Helsinki, and approval was obtained from the Institutional Review Board of Toyonaka Municipal Hospital (27 January 2020, No.2019-03-03). The requirement for informed consent was waived via the opt-out method on our hospital website.

\section{Colonoscopy procedure}

We routinely used an Olympus CF-Q260Al or CF-H290I series device (Olympus Optical Co., Tokyo, Japan) under conventional white light and narrow-band imaging. Carbon dioxide insufflation was used for all colonoscopies. If a patient preferred sedation, we used intravenous midazolam under oxygen saturation and electrocardiographic monitoring. Anticonvulsants, including scopolamine butyl bromide and glucagon, were used unless contraindicated. We detected colorectal polyps during colonoscopy, the indication for endoscopic resection (ER) was a polyp size $>5 \mathrm{~mm}$, and diminutive polyps were allowed to be followed up without resection based on the judgment of each endoscopist and in adherence to the Japan Gastroenterological Endoscopy Society (JGES) guideline [6]. However, diminutive flat and depressed lesions that were difficult to distinguish from adenoma or carcinoma were resected. The final decision to perform ER was made by each physician based on the bowel preparation and patient's condition. All removed lesions were diagnosed by full-time pathologists at our hospital.

\section{Definitions}

Cecum intubation was defined as the passage of the colonoscope tip from the rectum to the ileocecal valve. Each endoscopist evaluated the quality of bowel preparation using the Boston Bowel Preparation Scale (BBPS) [7].

In the present study, CRC was defined as submucosal and highly invasive cancer without ER indications. Advanced neoplasia was defined as intramural or slightly submucosal invasive cancer or adenoma with a diameter of at least $10 \mathrm{~mm}$ and highgrade dysplasia, villous or tubulovillous histologic characteristics, or any combination of these features [8]. The adenoma detection rate (ADR) and advanced neoplasia detection rate (ANDR) were defined as the proportion of colonoscopies that led to the removal of at least one histologically confirmed colorectal adenoma per colonoscopy. The polyp detection rate (PDR) was defined as the proportion of colonoscopies that led to the detection of at least one polyp without histological confirmation.

\section{Endpoints}

The primary endpoint was the incidence of colorectal neoplastic lesions (CRC and advanced neoplasia) in the diverticulitis group and the FIT-positive group. The secondary endpoints were the ADR and PDR in the two groups.

\section{Statistical analysis}

All continuous variables are presented as the mean \pm standard deviation (SD). Categorical variables are expressed as frequencies and percentages. The differences in categorical variables were evaluated for statistical significance by t-tests, and differences in continuous variables were evaluated with the Fisher's exact tests.

We stratified patients in both groups by age: under 40 years, 40 to 59 years, and 60 years or older. Then, we performed propensity score matching to minimize the potential confounding variables and selection biases. Age and sex were used as matching variables between the two groups, and we created a 1:1 matched cohort with an alpha of 0.05 to minimize the impact of potential selection bias. All calculated $P$ values were two-sided, and $P<0.05$ was considered statistically significant. All statistical analyses were performed with JMP statistical software (ver. 14.3, SAS Institute Inc., Cary, North Carolina, United States). 
Patients with suspected colonic diverticulitis, $\mathrm{N}=297$

\begin{tabular}{|l} 
Excluded, $\mathbf{N}=\mathbf{1 5}$ \\
- No definitive diagnosis, $\mathrm{N}=9$ \\
- Small intestine diverticulitis, $\mathrm{N}=1$ \\
- Ileus, $\mathrm{N}=1$ \\
- Fallopian tube cyst, $\mathrm{N}=1$ \\
- Enteritis, $\mathrm{N}=3$
\end{tabular}

Patients with a definitive diagnosis of colonic diverticulitis, $\mathrm{N}=282$

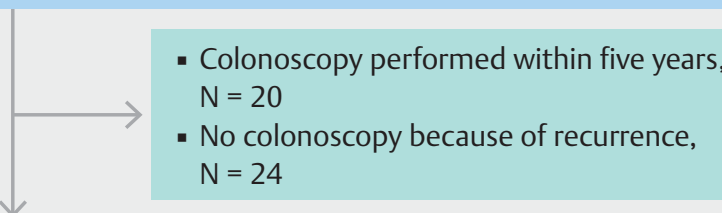

Patients with diverticulitis other than the above, $N=238$

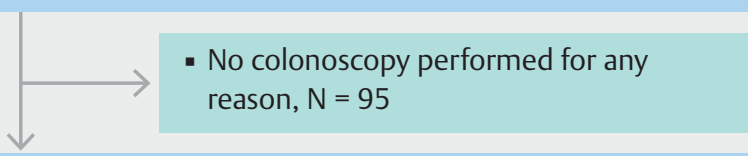

Patients enrolled in the diverticulitis group, $\mathrm{N}=143$
Patients who underwent colonoscopy because they were FIT positive, $\mathrm{N}=4232$

- No initial colonoscopy, N = 399

Patients who underwent an initial colonoscopy, $\mathrm{N}=3833$

Missing available data, $\mathrm{N}=2014$

Patients enrolled in the FIT-positive group, $\mathrm{N}=1819$

- Fig. 1 Study flowchart for the diverticulitis and FIT-positive groups.

\section{Results}

\section{Patients}

During the study period, 282 patients were finally diagnosed with colonic diverticulitis, except for 15 patients diagnosed with other diseases ( $\mathbf{F i g . 1}$ ). Acute diverticulitis was treated with bowel rest and antibiotics, but 36 patients required surgery. Of the patients with colonic diverticulitis, we excluded 139 patients with a previous colonoscopy within 5 years, unexamined colonoscopy results because of diverticulitis recurrence or other reasons, or unwillingness to undergo colonoscopy. Finally, 143 patients (60\%) underwent colonoscopy. Of them, four surgical patients underwent colonoscopy over 24 weeks. During the same study period, 4232 patients underwent colonoscopy because of positive FIT results. We excluded 399 patients due to a history of previous colonoscopy and 2014 patients due to unavailable data ( $\triangleright$ Fig.1). Finally, we enrolled 143 patients in the diverticulitis group and 1891 patients in the FIT-positive group as controls.

\section{Patient characteristics and colonoscopy quality indicators in patients with acute diverticulitis and positive FIT results}

- Table 1 shows the patient characteristics and quality indicators of the colonoscopies. The diverticulitis group was significantly younger than the FIT-positive group ( $53 \pm 15$ vs. $67 \pm 12$ years, $P<0.0001)$, but there was no significant sex difference between groups (male, $56 \%$ vs. $59 \%$ ). The sedative midazolam was more commonly used in the diverticulitis group. The average BBPS score, caecum intubation rate, intubation time, and total procedure time did not significantly differ between the two groups. Compared to the diverticulitis group, the FIT-positive group had a significantly higher number of detected polyps (1.0 vs $2.7, P<0.0001$ ), a higher CRC rate (0 vs $2.7 \%$, $P=0.0061)$, ANDR (5.6 vs. $14.0 \%, P=0.0017)$, ADR (19.6 vs. $53.2 \%, P<.0001)$, and PDR (44.1 vs. $91.0 \%, P<.0001)$. There were no CRC patients in the diverticulitis group ( $\triangleright$ Table 1 ).

\section{Detection rate of colorectal neoplasia according to age group}

Because CRC rates increase with age, we divided the patients into three groups according to age: under the age of 40 years, 40 to 59 years, and 60 years or older. $>$ Table 2 shows the number of patients and colorectal neoplasia detection rate of each age group. There were no significant sex differences between the diverticulitis and FIT-positive groups at any age. The CRC detection rates and ANDRs did not differ between the two groups at any age, but there were higher rates in the FIT-positive group than in the diverticulitis group, although there were no CRC patients under the age of 40 in either group. The ADR and PDR were significantly higher in the FIT-positive group than in the diverticulitis group at all ages. 
- Table 1 Baseline patient characteristics of the diverticulitis and FIT-positive groups and their quality indicators.

\begin{tabular}{|c|c|c|c|}
\hline & Diverticulitis & FIT-positive & $P$ value \\
\hline $\mathrm{N}$ & 143 & 1819 & \\
\hline Age mean $\pm S D$ & $53.1 \pm 15.3$ & $67.3 \pm 11.7$ & $<0.0001$ \\
\hline Sex, male (\%) & 56 & 59 & 0.5354 \\
\hline Sedative drug use ${ }^{1}$, N (\%) & $88(76.5)^{2}$ & $924(55.1)^{2}$ & $<0.0001$ \\
\hline BBPS score, mean \pm SD & $7.8 \pm 1.3$ & $7.5 \pm 1.5$ & 0.0528 \\
\hline Caecum intubation rate, $\mathrm{N}(\%)$ & $143(100)$ & $1683(98.5)^{2}$ & 0.1388 \\
\hline Intubation time, min & 9.3 & 9.8 & 0.4222 \\
\hline Total procedure time, min & 22.2 & 27.9 & $<0.0001$ \\
\hline Total number of detected polyps & $1.0 \pm 1.6$ & $2.7 \pm 2.6$ & $<0.0001$ \\
\hline Total number of resected polyps & $0.4 \pm 0.9$ & $1.2 \pm 1.6$ & $<0.0001$ \\
\hline $\begin{array}{l}\text { CRC, N } \\
(\%, 95 \% \mathrm{Cl})\end{array}$ & $\begin{array}{l}0 \\
(0)\end{array}$ & $\begin{array}{l}49 \\
(2.7,2.0-3.5)\end{array}$ & 0.0061 \\
\hline $\begin{array}{l}\text { ANDR, N } \\
(\%, 95 \% \mathrm{Cl})\end{array}$ & $\begin{array}{l}8 \\
(5.6,2.9-10.7)\end{array}$ & $\begin{array}{l}254 \\
(14.0,12.4-15.6)\end{array}$ & 0.0017 \\
\hline $\begin{array}{l}\text { ADR, N } \\
(\%, 95 \% \mathrm{Cl})\end{array}$ & $\begin{array}{l}28 \\
(19.6,13.9-26.8)\end{array}$ & $\begin{array}{l}968 \\
(53.2,50.9-55.5)\end{array}$ & $<0.0001$ \\
\hline $\begin{array}{l}\text { PDR, N } \\
(\%, 95 \% \mathrm{Cl})\end{array}$ & $\begin{array}{l}63 \\
(44.1,36.2-52.2)\end{array}$ & $\begin{array}{l}1655 \\
(91.0,89.6-92.2)\end{array}$ & $<0.0001$ \\
\hline
\end{tabular}

\section{Propensity score matching}

We performed propensity score matching analysis using age and sex because male sex as well as old age are strongly correlated with colorectal neoplasia [9]. After propensity score matching, we analyzed a total of 276 age- and sex-matched patients in both groups ( $>$ Table 3 ). There was no significant difference in the detection rates for CRC ( $0 \%$ vs. $0.7 \%, P=0.3164)$ or advanced neoplasia detection ( $5.8 \%$ vs $7.3 \%, P=0.6259)$ between the diverticulitis group and the FIT-positive group. The ADR and PDR were significantly higher in the FIT-positive group than in the diverticulitis group $(20.3 \%$ vs $43.5 \%, P<0.0001$; $45.7 \%$ vs $86.2 \%, P<0.0001)$.

\section{Discussion}

We found that the CRC rate after acute diverticulitis was not as high as that in asymptomatic patients with positive FIT results detected by a national CRC screening program in Japan. Propensity matching analysis after matching for sex and age also revealed that there was no significant difference in the CRC rate and ANDR between groups, but the ADR and PDR were significantly higher in the FIT-positive group. These results may suggest that colonoscopy can be recommended after colonic diverticulitis for CRC detection, as it is after positive FIT results, but does not seem to have as much impact after colonic diver- ticulitis as after positive FIT results regarding adenoma or polyp detection.

There have been reports on the necessity of colonoscopy to exclude CRC after colonic diverticulitis. In a systematic review reported by Sai et al., $2.1 \%$ of colonic diverticulitis patients diagnosed by CT who underwent surgery (14/771) were found to have CRC by barium enema or colonoscopy within 24 weeks. The authors concluded that CRC is more common in patients with colon diverticulitis than in the general population [10]. The highest incidence of CRC after diverticulitis was reported to be $7.4 \%(11 / 147)$ by Choi et al. [11]. The lowest value was $0.25 \%$ (1/404) by Lecleire et al. [12]. They concluded that the adenoma and CRC rates for colonoscopy after acute diverticulitis were similar to those for the screening program [12]. The incidence rates differ among reports. It has been questioned how essential colonoscopy is after diverticulitis.

A recent study recommended selecting patients with CRC risk factors for colonoscopy because the risk for CRC increases with older age, male sex, or family history [13-16]. Chan DKH et al. reported that among patients with diverticulitis under 50 years old who underwent colonoscopy, CRC or advanced adenoma were not observed in the younger patients [17]. Therefore, the authors concluded that follow-up colonoscopy after diverticulitis might not be necessary for younger patients [17]. In the present study, we evaluated the incidence of CRC among sex-matched groups stratified by age. There were no patients 
- Table 2 Number of patients and colorectal tumor detection rate for each age group.

\begin{tabular}{|c|c|c|c|c|}
\hline & & Diverticulitis & FIT-positive & $P$ value \\
\hline Age, years & Colorectal neoplasia & $N=143$ & $N=1819$ & \\
\hline \multirow[t]{6}{*}{$<40$} & N & $32(22.3)$ & $30(1.6)$ & \\
\hline & Male sex (\%) & $15(46.9)$ & $18(60.0)$ & 0.8017 \\
\hline & CRC, N (\%) & $0(0)$ & $0(0)$ & 0.0000 \\
\hline & ANDR, N (\%) & $0(0)$ & $3(10.0)$ & 0.0667 \\
\hline & ADR, N (\%) & $2(6.3)$ & $13(43.3)$ & 0.0007 \\
\hline & PDR, N (\%) & $6(18.8)$ & $24(80.0)$ & $<0.0001$ \\
\hline \multirow[t]{6}{*}{40 to 59} & N & $65(45.5)$ & $382(21.0)$ & \\
\hline & Male sex (\%) & $38(58.5)$ & $243(63.6)$ & 0.4877 \\
\hline & CRC, N (\%) & $0(0)$ & $6(1.6)$ & 0.3090 \\
\hline & ANDR, N (\%) & $5(7.7)$ & $38(10.0)$ & 0.5686 \\
\hline & ADR, N (\%) & $16(24.6)$ & $192(50.3)$ & 0.0001 \\
\hline & PDR, N (\%) & $28(43.1)$ & $335(87.7)$ & $<0.0001$ \\
\hline \multirow[t]{6}{*}{$\geq 60$} & N & $46(32.2)$ & $1407(77.4)$ & \\
\hline & Male sex (\%) & $24(52.2)$ & $805(57.2)$ & 0.5460 \\
\hline & CRC, N (\%) & $0(0)$ & $43(3.1)$ & 0.2287 \\
\hline & ANDR, N (\%) & $3(6.5)$ & $213(15.1)$ & 0.1060 \\
\hline & ADR, N (\%) & $10(21.7)$ & $763(54.2)$ & $<0.0001$ \\
\hline & PDR, N (\%) & $29(63.0)$ & $1296(92.1)$ & $<0.0001$ \\
\hline
\end{tabular}

FIT, fecal immunochemical test; CRC, colorectal cancer; ANDR, advanced neoplasia detection rate; ADR, adenoma detection rate; PDR, polyp detection rate.

under 40 years of age with CRC or advanced neoplasia in the diverticulitis group, and the ANDR tended to be higher in the FITpositive group, but the difference was not significant. For patients aged 40 years or older, the CRC detection rate and ANDR were not significantly different between the two groups. Colonic diverticulosis is an age-dependent disease, but diverticulitis was more common in younger patients than diverticular bleeding [18]. In the present study, patients under 40 years of age were more common in the diverticulitis group than in the FIT-positive group. Therefore, this result could support that colonoscopy may not always be necessary after diverticulitis for patients aged under 40 years if they do not have CRC risk factors.

Colorectal screening with a chemical fecal occult blood test (FOBT) can suppress CRC-related mortality, which has been demonstrated by several randomized controlled trials on screening [13-16]. Compared with FOBT, FIT has the advantage of not being affected by diet [19]. Therefore, FIT is currently used for CRC screening in Japan. The rate of CRC detection by colonoscopy after a positive FIT result is $3.9 \%$ to $4.2 \%$ [13-16]. In the present study, we defined patients with positive FIT results as the control group, because it is difficult to use screening colonoscopy data for asymptomatic populations in Japan who are not covered by the insurance system. Colonoscopy for FIT-positive patients aged 40 years or over is common and acceptable in Japan because the CRC screening program is offered to the population aged 40 years or over. Therefore, we think these patients are reasonable as a control group.

There is a strength in the present study. We evaluated the ADR in addition to the CRC and advanced neoplasia rates. Colorectal adenoma is a well-established precursor lesion for the majority of CRCs. Until now, there has been only one report analyzing the incidence of adenoma in patients with acute diverticulitis [12] that showed that there was no significant difference in ADR between acute diverticulitis patients with a mean of 61 years and control patients with a mean of 61 years who underwent screening colonoscopy (12.1 vs $14.6 \%, P=$ $0.35)$. Although the mean age of our diverticulitis group was younger (53 years) than that of this cohort, the ADR in the diverticulitis group was significantly lower than that in the FITpositive group (19.6 vs $53.2 \%, P<0.0001$ ). After the propensity score matching analysis using age and sex, the ADRs and PDRs, which were surrogate markers, in the diverticulitis group were approximately half those in the FIT-positive group.

There are several limitations to the current study. First, this study has a single-center retrospective design. Our cohorts could represent real-world data in Japan because our hospital is a regional core municipal hospital with 613 beds, and we analyzed consecutive patients with diverticulitis. Second, the diverticulitis group had a small sample size, which could have re- 
- Table 3 Patient characteristics and colorectal neoplasm detection rate after propensity score matching.

\begin{tabular}{|c|c|c|c|}
\hline & Diverticulitis & FIT-positive & $P$ value \\
\hline $\mathrm{N}$ & 138 & 138 & \\
\hline Age & 54 & 54 & 0.99 \\
\hline Sex, male (\%) & 56 & 57 & 0.81 \\
\hline Sedative drug use ${ }^{1}, \mathrm{~N}(\%)$ & $84(60.9)$ & $75(54.3)$ & 0.0023 \\
\hline BBPS sore, mean \pm SD & $7.8 \pm 1.3$ & $7.6 \pm 1.5$ & 0.2337 \\
\hline Caecum intubation rate, $\mathrm{N}(\%)$ & $138(100)$ & $138(100)$ & \\
\hline Intubation time, min & $9.3 \pm 6.6$ & $11.0 \pm 9.7$ & 0.4409 \\
\hline Total procedure time, min & $22.2 \pm 8.9$ & $27.9 \pm 14.4$ & 0.0041 \\
\hline Total number of detected polyps & $1 \pm 1.6$ & $1.9 \pm 1.8$ & $<0.0001$ \\
\hline Total number of resected polyps & $0.4 \pm 0.9$ & $1 \pm 1.5$ & $<0.0001$ \\
\hline $\begin{array}{l}\text { Colorectal cancer, } N \\
(\%, 95 \% \mathrm{Cl})\end{array}$ & $\begin{array}{l}0 \\
(0)\end{array}$ & $\begin{array}{l}1 \\
(0.7,0.1-4.0)\end{array}$ & 0.3164 \\
\hline $\begin{array}{l}\text { Advanced neoplasia, } \mathrm{N} \\
(\%, 95 \% \mathrm{Cl})\end{array}$ & $\begin{array}{l}8 \\
(5.8,3.0-11.0)\end{array}$ & $\begin{array}{l}10 \\
(7.3,4.0-12.8)\end{array}$ & 0.6259 \\
\hline $\begin{array}{l}\text { ADR, N } \\
(\%, 95 \% \mathrm{Cl})\end{array}$ & $\begin{array}{l}28 \\
(20.3,14.4-27.8)\end{array}$ & $\begin{array}{l}60 \\
(43.5,35.5-51.8)\end{array}$ & $<0.0001$ \\
\hline $\begin{array}{l}\text { PDR, N } \\
(\%, 95 \% \mathrm{Cl})\end{array}$ & $\begin{array}{l}63 \\
(45.7,37.6-54.0)\end{array}$ & $\begin{array}{l}119 \\
(86.2,79.5-91.0)\end{array}$ & $<0.0001$ \\
\hline
\end{tabular}

sulted in the absence of CRC patients in this group in the present study. Consequently, this study was underpowered to show a difference in CRC incidences between patients with positive FITs and those with diverticulitis. Therefore, we evaluated adenoma, which can be a surrogate marker for CRC. Third, almost half of the patients with positive FIT results during the study period were excluded due to missing or unavailable data, which may cause bias. However, we were able to enroll 1819 FIT-positive patients, which we considered a sufficient number for the control group to compare with the diverticulitis group because of the very narrow $95 \%$ confidential intervals in CRC rate, ANDR, ADR, and PDR. Finally, we did not consider the patient history of other cancers, family history of CRC, or disease sidedness, all of which could have affected the results.

\section{Conclusion}

We found that patients with acute diverticulitis and those with positive FIT results had similar CRC rates and ANDRs, but those with acute diverticulitis had significantly lower ADRs and PDRs. It may not be necessary to perform subsequent colonoscopy to exclude malignancy for patients with colonic diverticulitis diagnosed by cross-sectional imaging methods such as CT.

\section{Competing interests}

The authors declare that they have no conflict of interest.

\section{References}

[1] Stollman N, Smalley W, Hirano I et al. American Gastroenterological Association Institute Guideline on the Management of Acute Diverticulitis. Gastroenterology 2015; 149: 1944-1949

[2] Strate LL, Peery AF, Neumann I. American Gastroenterological Association Institute Technical Review on the Management of Acute Diverticulitis. Gastroenterology 2015; 149: 1950-1976, e1912

[3] Nagata N, Ishii N, Manabe N et al. Guidelines for Colonic Diverticular Bleeding and Colonic Diverticulitis: Japan Gastroenterological Association. Digestion 2019; 99: (Suppl. 01): 1-26

[4] Daniels L, Unlu C, de Wijkerslooth TR et al. Routine colonoscopy after left-sided acute uncomplicated diverticulitis: a systematic review. Gastrointest Endosc 2014; 79: 378-389; quiz 498-498 e375

[5] Granlund J, Svensson T, Granath F et al. Diverticular disease and the risk of colon cancer - a population-based case-control study. Aliment Pharmacol Therap 2011; 34: 675-681

[6] Tanaka S, Kashida H, Saito Y et al. JGES guidelines for colorectal endoscopic submucosal dissection/endoscopic mucosal resection. Digest Endosc 2015; 27: 417-434

[7] Lai EJ, Calderwood AH, Doros G et al. The Boston bowel preparation scale: a valid and reliable instrument for colonoscopy-oriented research. Gastrointest Endosc 2009; 69: 620-625 
[8] Lieberman DA, Weiss DG, Bond JH et al. Use of colonoscopy to screen asymptomatic adults for colorectal cancer. Veterans Affairs Cooperative Study Group 380. N Engl J Med 2000; 343: 162-168

[9] Amano T, Nishida T, Shimakoshi $\mathrm{H}$ et al. Number of polyps detected is a useful indicator of quality of clinical colonoscopy. Endosc Int Open 2018; 6: E878-E884

[10] Zaman S, Chapman W, Mohammed I et al. Patients with computed tomography-proven acute diverticulitis require follow-up to exclude colorectal cancer. Intest Res 2017; 15: 195-202

[11] Choi YH, Koh SJ, Kim JW et al. Do we need colonoscopy following acute diverticulitis detected on computed tomography to exclude colorectal malignancy? Digest Dis Sci 2014; 59: 2236-2242

[12] Lecleire S, Nahon S, Alatawi A et al. Diagnostic impact of routine colonoscopy following acute diverticulitis: A multicenter study in 808 patients and controls. United Europ Gastroenterol J 2014; 2: 301-306

[13] Hardcastle JD, Chamberlain JO, Robinson MH et al. Randomised controlled trial of faecal-occult-blood screening for colorectal cancer. Lancet 1996; 348: 1472-1477
[14] Mandel JS, Bond JH, Church TR et al. Reducing mortality from colorectal cancer by screening for fecal occult blood. Minnesota Colon Cancer Control Study. N Engl J Med 1993; 328: 1365-1371

[15] Kronborg O, Fenger C, Olsen J et al. Randomised study of screening for colorectal cancer with faecal-occult-blood test. Lancet 1996; 348 : 1467-1471

[16] Mandel JS, Church TR, Bond JH et al. The effect of fecal occult-blood screening on the incidence of colorectal cancer. N Engl J Med 2000; 343: $1603-1607$

[17] Chan DKH, Tan KK. There Is no role for colonoscopy after diverticulitis among Asian patients less than 50 years of age. Gastrointest Tumors 2017; 3: 136-140

[18] Urabe M, Nishida T, Shimakoshi $\mathrm{H}$ et al. Distinct Clinical factors in hospitalized patients with diverticular bleeding and diverticulitis. Digestion 2019; 99: 239-246

[19] Saito H. Screening for colorectal cancer by immunochemical fecal occult blood testing. Jpn J Cancer Res 1996; 87: 1011-1024 